\title{
Large-scale, core-team assessed evaluation of the SAVE technique
}

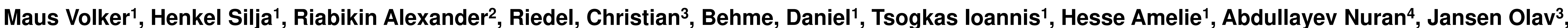
Wiesmann Martin², Mpotsaris Anastasios ${ }^{2}$, Psychogios Marios-Nikos ${ }^{1}$

${ }^{1}$ Department of Neuroradiology, University Medical Center Goettingen, Goettingen, Germany. ${ }^{2}$ Department of Neuroradiology, University Hospital Aachen, Aachen, Germany. ${ }^{3}$ Department of Neuroradiology, University Hospital Kiel, Kiel, Germany. ${ }^{4}$ Department of Radiology, University Hospital Cologne, Cologne, Germany

\section{Background and Purpose}

Development of new thrombectomy techniques and improvements of existing methods were evaluated to improve the rate of successful reperfusion and minimize the events of distal embolization in patients suffering from intracranial large vessel occlusion (LVO). The "Stent retriever Assisted Vacuum-locked Extraction" (SAVE) technique was recently described as a method for achieving favorable angiographic results concerning a high rate of successful and complete reperfusion and a low rate of downstream embolization based on the principle of a distal (stent retriever) and proximal (aspiration catheter) capture of the clot. The purpose of the present study is to report our ongoing experience with SAVE.

\section{Objective}

To assess the first-pass and overall complete and near-complete reperfusion, defined as a modified Thrombolysis in Cerebral Infarction (mTICl) score of $2 \mathrm{c}$ and 3. Furthermore, we wanted to assess the number of passes, time from groin puncture to reperfusion, embolization to new territories (ENT), and post-interventional symptomatic intracranial hemorrhage $(\mathrm{sICH})$.

\section{Methods}

A retrospective analysis of data was performed to identify all patients who consecutively underwent thrombectomy with SAVE at the participating centers between November 2015 and January 2018. All consecutive cases with LVO were included from center A (Goettingen, 95 cases), center B (Aachen, 42 cases), center C (Cologne, 38 cases), and center D (Kiel, 25 cases). Baseline, clinical, and angiographic parameters were extracted from a prospectively acquired database in center $A$ and $C$.

Inclusion criteria were clinical diagnosis of acute ischemic stroke, intention to perform endovascular stroke treatment (i.e. initiation of endovascular procedure) with proper angiographic documentation of the SAVE maneuver, and age $\geq 18$ years. Ischemic stroke was diagnosed with non-contrast CT (or FDCT in cases of one-stop management) and CTA (or multiphase FDCTA). All eligible patients received IVT.

All images from digital subtraction angiography were re-evaluated in accordance with the recommendations of the Cerebral Angiographic Revascularization Grading Collaborators from an internal core-team, which was blinded to the clinical outcome, presentation, or any demographic data to assess pre-interventional, initial state and extent of tissue reperfusion after each thrombectomy attempt.

SAVE technique: Based on a triaxial approach, access to cerebral vasculature is obtained with an 8 French $(F)$ guide catheter/long $8 F$ sheath distally in the internal carotid artery (ICA, Figure 1). A microcatheter is advanced through a $5 \mathrm{~F} / 6 \mathrm{~F}$ aspiration catheter past the occlusion site in an M2/A2 vessel under continuous aspiration or with the help of a 0.014 ' inch microwire. A stent retriever is advanced and placed primarily distally to and with the proximal third across the occlusion site by using the active push deployment technique. The stent retriever is deployed over 2-8 minutes. The microcatheter is slowly retracted in order to maximize the inner volume of the aspiration catheter. The aspiration catheter is then advanced to the proximal face of the clot and the aspiration pump is connected and activated. The stent retriever is gently retracted, while the tip of the aspiration catheter is further advanced until a wedge position is reached. The negative pressure within the aspiration catheter is then retained with the use of a $60 \mathrm{ml}$ vacuum syringe and the aspiration pump is connected to the guide catheter or long sheath. Stent retriever and aspiration catheter are then withdrawn into the guide catheter as a unit.

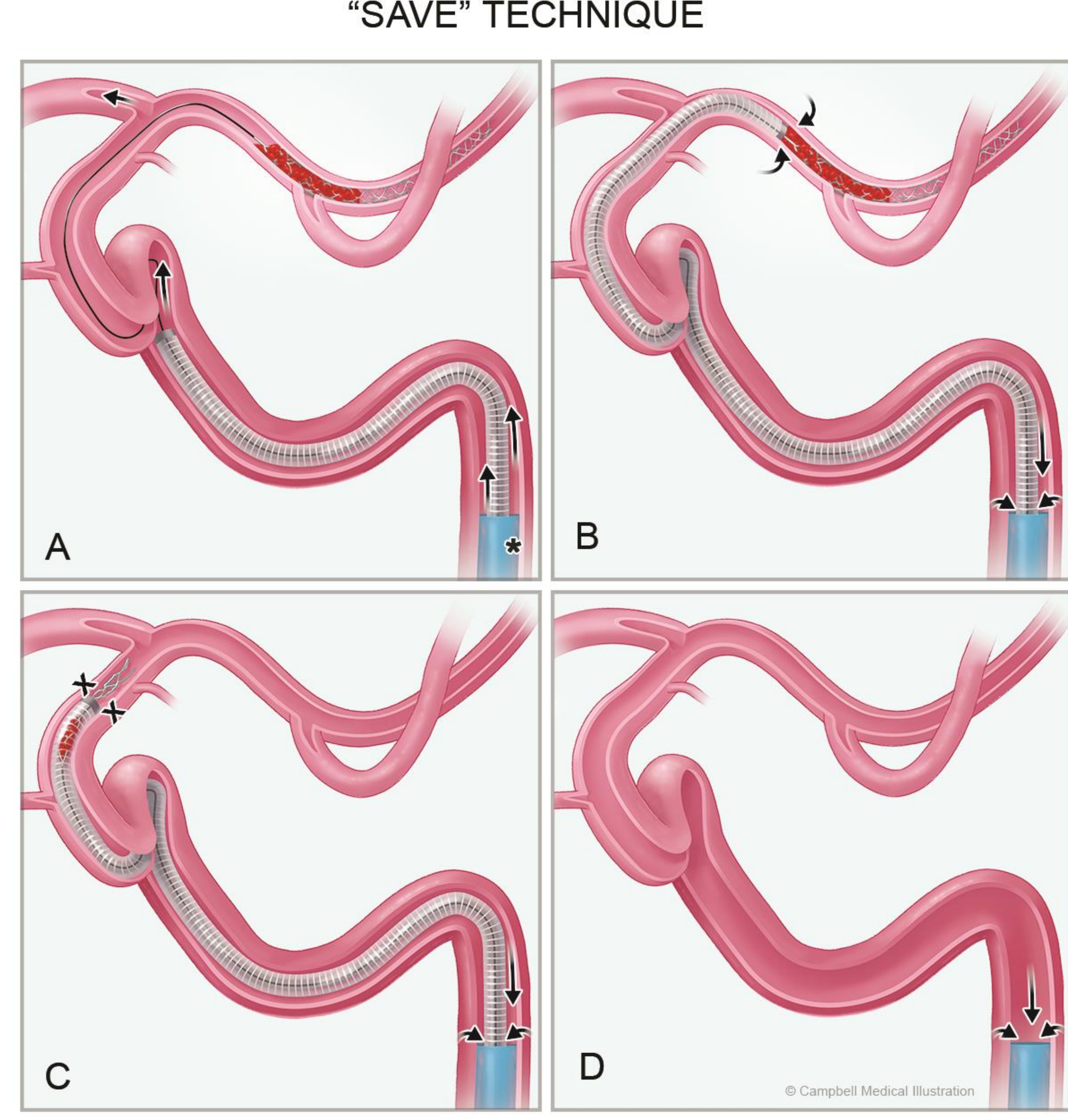

Figure 1: SAVE technique

\section{Results}

Median age was 78 years (IQR 68-85) and 78 patients were male (39\%). The median NIHSS at admission was 16 (IQR 12-20) and rate of IVT was 58\%. Occlusion sites were: ICA-T in 39/200 (19.5\%), M1 in 126/200 (63\%), M2 in 30/200 (15\%), and others in 5/200 (2.5\%) cases. First-pass complete/near-complete reperfusion ( $\mathrm{mTICI} \geq 2 \mathrm{c}$ ) was achieved in 114/200 (57\%) with complete reperfusion ( $\mathrm{mTICl} 3$ ) in 90 patients $(45 \%)$. An overall rate of $\mathrm{mTICl} 2 \mathrm{c}$ and 3 on final angiogram was reached in 154 patients (77\%) with 111 individuals $(56 \%)$ completely reperfused $(\mathrm{mTICl} 3)$. The rate of successful reperfusion ( $\mathrm{mTICl} \geq 2 \mathrm{~b}$ ) after one attempt was $74 \%$ and $95 \%$ at the end of the procedure with a median of 1 (IQR 1-2) attempt and a maximum of six passes. The overall median time from groin puncture to reperfusion was $34 \mathrm{~min}$ (IQR 25-52). ENTs were observed in 3 patients $(1.5 \%)$ and the rate of $\mathrm{SICH}$ was $2.6 \%$. Median NIHSS at discharge was 4 (IQR 2-11).

A subgroup analysis investigating the angiographic results with respect to the occlusion site revealed that the use of SAVE tended to be more effective in M1 occlusions compared to ICA-T occlusions in terms of complete reperfusion after a single pass (66/126 (52\%) vs. $10 / 39(26 \%), \quad p=0.084)$ and complete/near-complete reperfusion $(80 / 126(64 \%)$ vs. $15 / 39(38 \%), p=0.154)$. The final rate of complete reperfusion was higher in $\mathrm{M} 1$ vs. ICA-T occlusions $(61 \%$ vs. $49 \%$, $\mathrm{p}=0.538$ ). The median time from groin puncture to reperfusion was significantly faster in patients suffering from M1 occlusions (32 minutes) compared to ICA-T occlusions (51 minutes, $p<0.001)$ with a median number of one pass (M1) vs. two passes (ICA-T)

\section{Conclusions}

- Mechanical thrombectomy using the SAVE technique is effective, fast and secure.

- SAVE leads to high-rates of complete/near-complete reperfusion.

- SAVE tended to be more effective in M1 occlusions compared to ICA-T occlusions

We recommend SAVE as first-line therapy in patients with intracranial LVO. 